Article

\title{
Thermal Comfort-Based Personalized Models with Non-Intrusive Sensing Technique in Office Buildings
}

\author{
Siliang Lu ${ }^{1} * \mathbb{C}$, Weilong Wang ${ }^{2}$, Shihan Wang ${ }^{3}$ and Erica Cochran Hameen ${ }^{1}$ \\ School of Architecture, Carnegie Mellon University, Pittsburgh, PA 15217, USA; ericac@andrew.cmu.edu \\ 2 Krannert School of Management, Purdue University, West Lafayette, IN 47907, USA; wang4167@purdue.edu \\ 3 Salesforce, 1st Francis PL, CA 94107, USA; wjason1115@gmail.com \\ * Correspondence: siliang1@andrew.cmu.edu
}

Received: 10 March 2019; Accepted: 24 April 2019; Published: 28 April 2019

Featured Application: The proposed thermal comfort-based personalized models can be applied into personalized heating and cooling system in open-plan office buildings.

\begin{abstract}
Heating, ventilation and air-conditioning (HVAC) systems play a key role in shaping the built environment. However, centralized HVAC systems cannot guarantee the provision of a comfortable thermal environment for everyone. Therefore, a personalized HVAC system that aims to adapt thermal preferences has drawn much more attention. Meanwhile, occupant-related factors like skin temperature have not had standardized measurement methods. Therefore, this paper proposes to use infrared thermography to develop individual thermal models to predict thermal sensations using three different feature sets with the random forest (RF) and support vector machine (SVM). The results have shown the correlation coefficients between clothing surface temperature and thermal sensation are $11 \%$ and $3 \%$ higher than those between skin temperature and thermal sensation of two subjects, respectively. With cross-validation, SVM with a linear kernel and penalty number of 1 , as well as RF with 50 trees and the maximum tree depth of 3 were selected as the model configurations. As a result, the model trained with the feature set, consisting of indoor air temperature, relative humidity, skin temperature and clothing surface temperature, and with linear kernel SVM has achieved 100\% recall score on test data of female subjects and $95 \%$ recall score on that of male subjects.
\end{abstract}

Keywords: adaptive thermal comfort; infrared thermography; support vector machine; random forest; intelligent workplace

\section{Introduction}

Most people spend $90 \%$ of their time indoors [1] since buildings can provide satisfactory environments for human beings. Indoor environment quality (IEQ) is always used to evaluate the quality of the built environments created with different building systems such as acoustic quality, air quality, spatial quality, visual quality and thermal quality. As one of the key building systems, Heating, ventilation and air-conditioning (HVAC) systems affect all of these qualities, particularly indoor air quality (IAQ) and indoor thermal quality in commercial buildings such as open-plan office buildings. Moreover, open-plan offices have become a trend nowadays since they increase communication between employees and reduce construction cost. However, open-plan office buildings are also faced with problems like unsatisfactory shared indoor temperature and humidity due to different occupants' thermal preferences. Therefore, many researchers have developed the advanced technologies for the open-plan workplace so as to improve individual thermal comfort. 


\subsection{Background of Thermal Models for HVAC System of Office Buildings}

As defined by ANSI/ASHRAE 55: Thermal Environmental Conditions for Human Occupancy [2] and ISO7730: Ergonomics of the thermal environment-Analytical determination and interpretation of thermal comfort using calculation of the PMV and PPD indices and local thermal comfort criteria [3], thermal comfort is the condition of mind that expresses satisfaction with the thermal environment and is assessed by subjective evaluation. Therefore, thermal comfort is the combined result of physical environment and psychological activities. In the course of thermal comfort theory, static thermal comfort and adaptive thermal comfort have become two main categories. In terms of static thermal comfort, predicted mean vote (PMV) developed by Fanger [4] is the most widely accepted thermal model where seven variables are accepted to be the indicators of thermal comfort, which are indoor air temperature, indoor relative humidity, indoor air velocity, mean radiant temperature, clothing insulation, metabolic rate and the external work (normally 0). However, PMV was derived from a strictly controlled climate chamber where the thermal environment is static and is different from the real office environment. Moreover, since PMV predicts the average vote of a large group of people based on the seven-point thermal sensation scale, instead of individual thermal comfort, it only describes the overall thermal sensation of multiple occupants in a shared thermal environment.

In order to overcome the disadvantages of static thermal comfort, adaptive thermal comfort aims to provide insights in increasing opportunities for personal and responsive control, thermal comfort enhancement, energy consumption reduction and climatically responsive and environmentally responsible building design [5]. One of the milestone projects of adaptive thermal comfort is the ASHRAE RP-884, which collected a total of 22,000 sets of data from the real office environments across the world. This project has been widely used to investigate various adaptive thermal models, which have been integrated into personalized HVAC controls. With RP884 dataset, Lee et al. [6] proposed a method for learning personalized thermal preference profiles by formulating a combined classification and inference problem with 5-cluster models. Moreover, the thermal preference of a new user is inferred by mixture of sub-models for each cluster where clusters are used to group occupants with similar thermal preferences. However, instead of predicting 7-point thermal sensation described in ASHRAE 55, the paper predicts thermal preferences with three classes, namely "want cooler", "want warmer" and "no change". Auffenberg et al. [7] proposed a personalized thermal model using a Bayesian network to learn individual thermal comfort by predicting thermal sensation of occupants within a specific area, such as San Francisco with ASHRAE RP-884 dataset. However, the results were not promising that the highest accuracy of the model was only $30 \%$. In addition to ASHRAE RP884 dataset, other data-driven studies have also been conducted for the development of adaptive thermal model comfort model. For instance, a unified framework was presented by Kim et al. [8] for personal comfort models and challenges were discussed for the application of personal comfort models in the real world. In the framework, instead of survey, the feedback regarding thermal preferences was collected with the interactions between personal a comfort system (PCS) and occupants. Moreover, machine learning algorithms were proposed for the development of personal comfort models.

Thermoregulation is a dynamic equilibrium with the environment where body regulates its internal core temperature in terms of heat generation and heat exchange with the environment [9]. In detail, if the ambient temperature is below skin temperature, heat is dissipated from the body by radiation and convection. On the other hand, if the skin temperature is lower than ambient temperature, heat will be absorbed by the body so that the skin temperature increases [10]. Even if heat exchange also occurs due to the metabolic process of the human body, since occupants usually sit or walk without too much metabolic heat production in office buildings, whether the individual is thermally comfortable or not is more related to skin temperature.

Motivated by thermoregulation theory, many researchers have proposed thermal models with the mean skin temperature or the most representative local skin temperature. Liu et al. [11] used the mean skin temperature of 10 measurement locations, including forehead, chest, upper arm, back, abdomen, elbow, hand, thigh, calf and foot to evaluate thermal comfort for a person in a sleeping 
posture under steady thermal environment. The results turned out that the mean skin temperature at the three different thermal comfort levels was statistically significant. In terms of local skin temperature, Choi [12] tested skin temperatures of 10 body locations to select the best location for thermal comfort inference, including forehead, upper arm, belly, wrist, hand, thigh, calf and foot. The results turned out that wrist was the most responsive area to infer thermal comfort with the lowest $p$-value. Moreover, Sim et al. [13] established multiple linear regression models to estimate thermal sensations with variables based on skin temperature. As a result, the models using temperatures of the fingertips and wrist showed the highest accuracy. Li et al. [14] also proposed multiple linear regression models to estimate thermal sensation under various active states using wrist skin temperature-related variables. The study indicated skin temperature, its time differential and heart rate could be used for estimating individual thermal sensation. Besides regression, thermal sensation prediction can also be seen as a classification problem where various classification algorithms can be implemented.

In order to investigate other statistical methods to establish thermal models besides regression, a recent research on thermal comfort inference utilized the wearable device of a pair of eyeglass with infrared (IR) arrays, and demonstrated that $82.8 \%$ of prediction accuracy for detection uncomfortable conditions was obtained with hidden Markov model (HMM) [15]. Moreover, Huang et al. [16] used wearable devices like wearable fitness trackers to measure physical movements, sweat level and skin temperature so as to infer individual thermal comfort with machine learning algorithms including random forest (RF) and support vector machine (SVM). In addition, Dai et al. [17] implemented SVM to predict thermal demands using skin temperature collected from various locations with wearable sensors. As a result, SVM classifiers with linear kernel were preferred to Gaussian kernel, which achieved over $90 \%$ accuracy. Based on the literature review, classification models have performed so well as regression models. However, instead of a 7-point thermal sensation scale, most of those models only predict thermal sensations with three classes, which are uncomfortably warm, comfortable and uncomfortably cool.

\subsection{Background of Sensing Technique for Developing Thermal Models in Office Buildings}

Besides different methods of thermal models, the development of an advanced sensing technique has also promoted the development of adaptive thermal comfort. The current sensing technique used for thermal comfort inference can be mainly divided into two categories-wearable sensing devices and contactless sensing devices. For wearable devices, wrist band has been the most popular one [11-20]. Moreover, Ghahramani et al. proposed a wearable infrared eyeglass frame to measure skin temperature at different points of the skin, including nose, front face, back of ear and cheek bone so as to infer thermal preferences [18].

Even if wearable devices can directly measure variables like skin temperature to indicate thermal comfort, the major disadvantage is intrusiveness. Therefore, contactless measurement methods have drawn much more attention nowadays, especially infrared (IR) thermography. IR thermography utilizes the emitted infrared radiation to measure surface temperature of an object. In order to measure surface temperature with IR thermography correctly, emissivity of the object has to be known since most of actual objects are grey bodies. Nowadays, IR thermography has been widespread and applied into various functions [21], such as building diagnostics [22], fault detection as well as thermal comfort analysis [23-26]. Ranjan and Scott [24] have used IR cameras to dynamically detect and predict thermal comfort. They classified thermal preferences based on skin temperature of forehead, cheek, jaws, upper neck, lower neck, palm core, palm and back of hand, and found that the face outperformed other body regions. Han et al. [25] utilized infrared imaging to measure skin temperature and control the indoor environment with self-learning algorithms. As a result, $98 \%$ of the occupants' feedback demonstrated the control system was able to achieve satisfactory thermal environments. Additionally, an empirical study utilized an infrared (IR) sensor called Lepton to estimate occupant thermal comfort level by measuring skin temperature measured from different face regions. The results have shown that ears, nose and cheeks are most indicative to thermal comfort [26]. 
To summarize, contactless sensing technique can solve the issue of intrusiveness while maintain high performance of thermal comfort inference. Moreover, the adaptive thermal comfort is more realistic to the actual indoor environment than static thermal comfort and the performance can be enhanced with machine learning algorithms. Therefore, developing adaptive thermal models with contactless sensing techniques have become an interesting research area recently.

\subsection{Research Contributions}

Based on a literature review of thermal comfort research, it can be concluded that the recent studies related to thermal comfort have been focused on the development of different sensing techniques to build individual thermal models and apply such models into occupant-responsive HVAC system [12-20,24-26]. Compared to sensing approaches like wearable devices, contactless vision-based occupancy sensors such as IR camera are much less intrusive and have drawn much more attention than before [24-26]. However, most recent studies using IR camera for thermal comfort use regression analysis with only skin temperature, which may not have high performance when predicting occupant thermal comfort. Therefore, taking both environment-related and occupant-related factors into account, this research aims to develop adaptive thermal models with three different feature sets trained with RF and SVM. In order to collect occupant data, a six day experimental study was conducted in an open-plan office in Shanghai with the non-intrusive sensing system consisting of indoor air temperature and relative humidity sensor and an IR camera. The study contributes to the field of the personalized thermal comfort by proposing new feature sets and prediction algorithms to develop adaptive thermal models, which could further be integrated into personalized system control as well as task ambient conditioning system in open-plan offices. The rest of the paper consists of methodology, including the experimental setup and the development of thermal models, result analysis and discussions about the prediction performances as well as the conclusion and future work.

\section{Methodology}

In this study, the development of the thermal models can be divided into two parts. One is the field study to collect data for the development of thermal models with the proposed algorithms and the other is the real-time thermal model predictions, as shown in Figure 1. The data collection was implemented in C++ with ESP8266 while the models were developed in Scikit learn with Python [27].

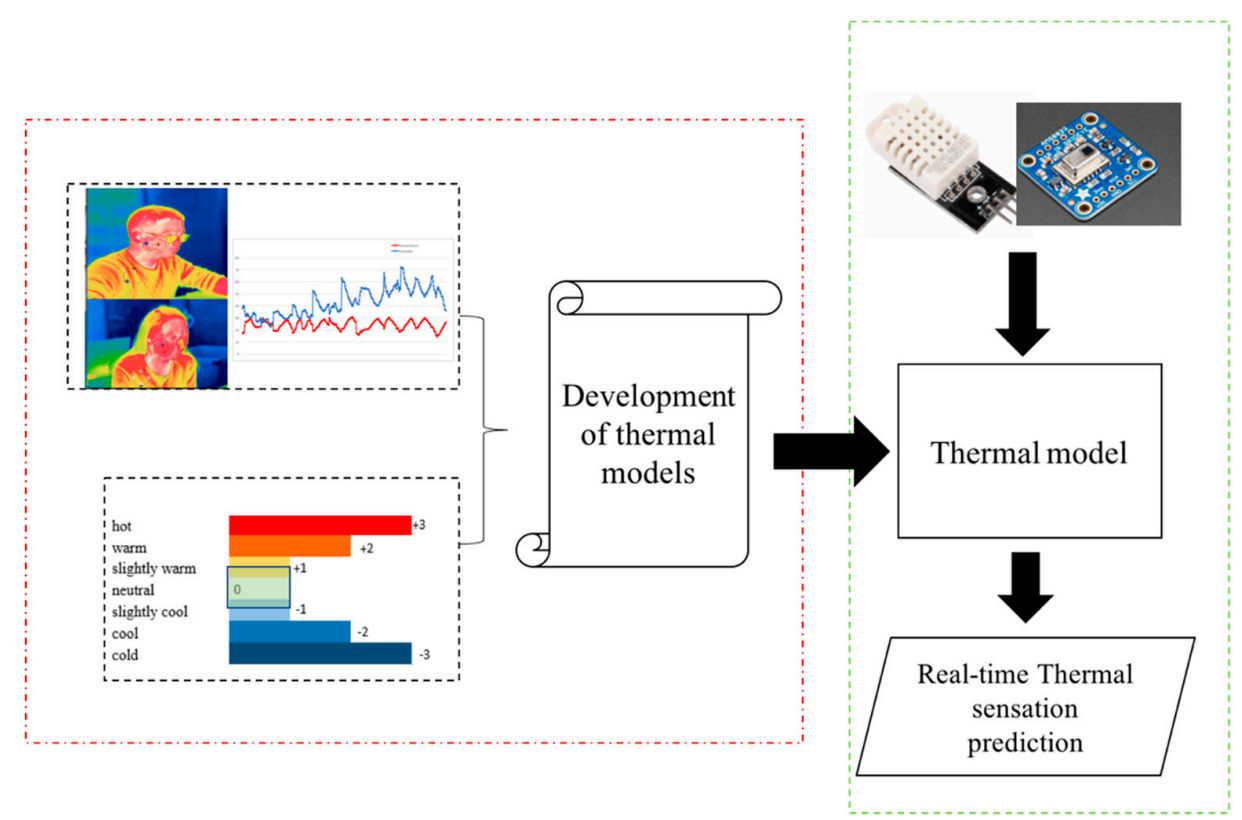

Figure 1. The over diagram of the proposed methodology. 


\subsection{Experimental Setup}

Since this study aims to develop individual thermal models which capture the fine-tuned differences between different people, instead of developing a generic thermal model based on large group of people, it makes more sense to develop individual thermal models. Moreover, for machine learning algorithms, the performance will increase with a greater amount of data. However, even if the common datasets like RP884 collected data from large group of participants, only less than 10 instances were collected for each individual. Therefore, unlike RP884, in this study, individual thermal models were developed based on a six-day experimental study to collect certain amount of data with two healthy subjects in their mid-twenties (one male and one female) between 3/11/2018 and 3/16/2018 in an office building in Tongii University, Shanghai.

Based on the design of non-intrusive sensing systems and a literature review of principle factors related to thermal comfort, the measured variables are shown in the following Table 1 . The interval of data collection is five minutes, which corresponds with the survey responses. The experiment used the IR camera to measure the side face skin temperature and clothing surface temperature by manually selecting the face area and clothing area in the high-resolution thermal images, and then calculating the average surface temperature within those areas in FLIR tools, respectively. Figure 2 shows the participants' status in the experimental study and the thermographic photos, respectively. Before each session, the participants' clothes were reported, and heart rates were measured by a free mobile app called Instant Heart Rate so as to ensure the participants have similar thermal status in the beginning. Lastly, clothing insulation was manually estimated based on ASHRAE 55 and was used for calculating PMV values. In order to get the accurate skin temperature, the emissivity was set to be 0.98 .

Table 1. Measured variables and sensor tools.

\begin{tabular}{lccc}
\hline \multicolumn{1}{c}{ Variable } & Sensor Tool & Resolution & Operating Range \\
\hline Skin temperature $\left({ }^{\circ} \mathrm{C}\right)$ & FLIR B8400 & $320 \times 240$ pixels & $-20^{\circ} \mathrm{C} \sim 120^{\circ} \mathrm{C}$ \\
Clothing surface temperature $\left({ }^{\circ} \mathrm{C}\right)$ & FLIR B8400 & $320 \times 240$ pixels & $-20^{\circ} \mathrm{C} \sim 120^{\circ} \mathrm{C}$ \\
Indoor air temperature $\left({ }^{\circ} \mathrm{C}\right)$ & DHT22 & $0.1^{\circ} \mathrm{C}$ & $-4{ }^{\circ} \mathrm{C}-80^{\circ} \mathrm{C}$ \\
Indoor relative humidity $(\%)$ & DHT22 & $0.1^{\circ} \mathrm{C}$ & $-40^{\circ} \mathrm{C}-80^{\circ} \mathrm{C}$ \\
Clothing insulation (clo) & Manually identify the clothing type and & \\
& find the insulation values based on & \\
\hline
\end{tabular}

FLIR B8400: infrared camera; DHT22: temperature \& humidity sensor.

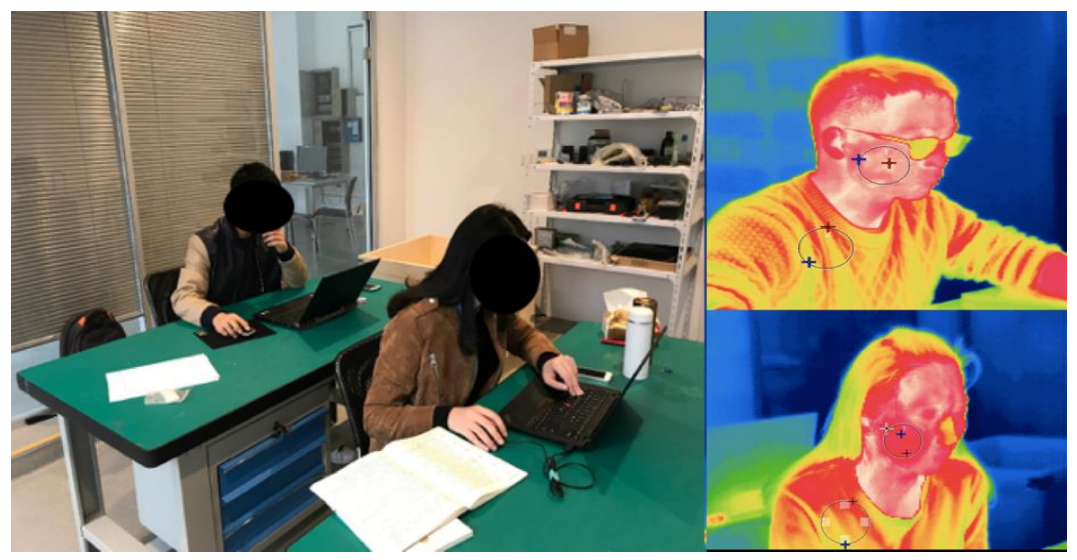

Figure 2. The RGB and IR images of the two subjects.

Even if neutral sensation doesn't necessarily mean thermal comfort, it is a precondition of comfort [28]. Therefore, the statement that an occupant feels thermally comfortable when they feel the thermal environment is neutral was utilized in this study, and a 7-point thermal sensation scale as a classification label was used, as shown in Table 2. 
Table 2. ASHRAE Thermal-sensation scale.

\begin{tabular}{cc}
\hline Thermal Sensation Vote (TSV) & Description \\
\hline-3 & very cold \\
-2 & cold \\
-1 & cool \\
0 & neutral \\
1 & warm \\
2 & hot \\
3 & very hot \\
\hline
\end{tabular}

In order to create different thermal environment conditions, an overhead air conditioner and four convective heaters were controlled in transient conditions where the air temperature increases no more than $0.2^{\circ} \mathrm{C} / \mathrm{min}$ on average, as shown Figure 3. Moreover, Figure 4 shows the floorplan of the testbed where two subjects were seated in two separate desks and the camera has two locations to take photos of each subject, respectively.

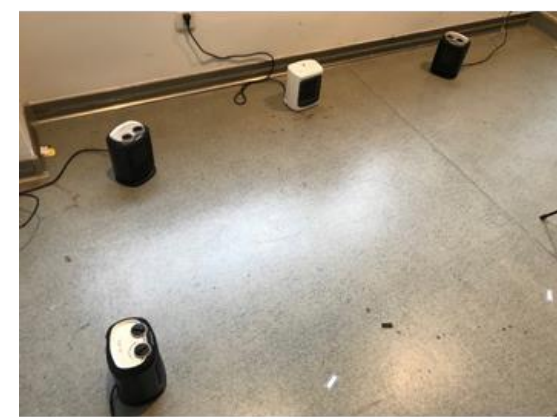

(a)

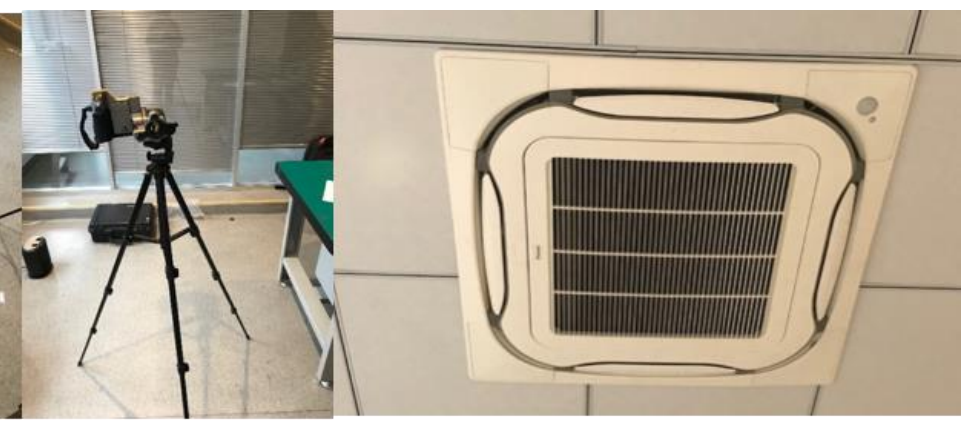

(b) (c)

Figure 3. Experimental Setup. (a) Heaters, (b) FLIR B400 IR camera, (c) Air-conditioning terminal.

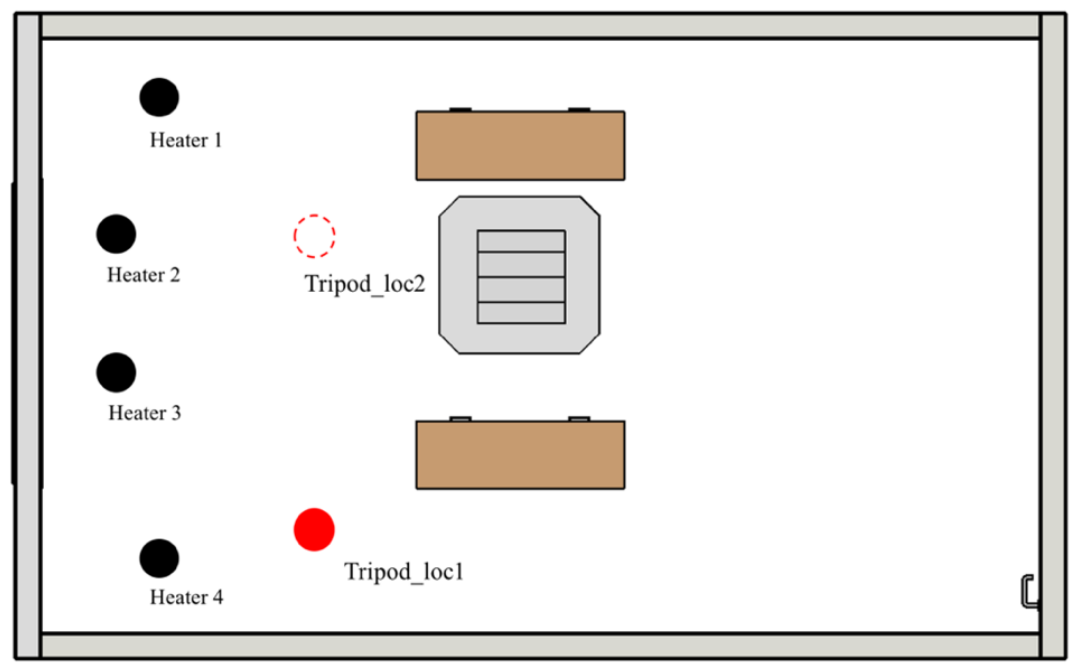

Figure 4. Floorplan of the test bed.

To avoid discomfort due to high air velocity and asymmetric radiation, the participants were asked to adjust the locations of their working areas if they could feel a draft or if they could feel the sunlight before measurement. In addition, there were a total of 14 different sessions and each session lasted for at least two hours. Table 3 shows the temperature changes during each session. Since the participants attended the study for all sessions, all levels of thermal sensations were reported at least once by participants even if the majority votes belonged to neutral sensation. 
Table 3. Temperature changes during each session.

\begin{tabular}{ccccccc}
\hline Date & Start Time & Transition Time & End Time & $\begin{array}{c}\text { Start } \\
\text { Temperature } \\
\left({ }^{\circ} \mathbf{C}\right)\end{array}$ & $\begin{array}{c}\text { Transitional } \\
\text { Temperature } \\
\left({ }^{\circ} \mathbf{C}\right)\end{array}$ & $\begin{array}{c}\text { End } \\
\text { Temperature } \\
\left({ }^{\circ} \mathbf{C}\right)\end{array}$ \\
\hline \multirow{3}{*}{$3 / 11$} & $9: 50$ & $11: 00$ & $11: 50$ & 17.4 & 29.3 & 22.3 \\
& $13: 45$ & $15: 20$ & $16: 45$ & 22.6 & 29 & 24.3 \\
& $19: 30$ & $21: 45$ & $22: 30$ & 19.3 & 28.5 & 20.9 \\
\hline \multirow{2}{*}{$3 / 12$} & $10: 10$ & $11: 10$ & $11: 30$ & 17.7 & 29.5 & 25.3 \\
& $13: 40$ & $14: 55$ & $15: 30$ & 21.2 & 28.4 & 24.4 \\
\hline $3 / 13$ & $9: 35$ & $11: 20$ & $12: 00$ & 19 & 28.9 & 24.1 \\
& $13: 40$ & & $17: 30$ & 29.9 & & 18.5 \\
$3 / 14$ & $19: 40$ & $21: 05$ & $22: 35$ & 21.2 & 28.5 & 24.3 \\
\hline \multirow{2}{*}{$3 / 15$} & $9: 30$ & $10: 50$ & $11: 35$ & 20.8 & 29 & 26.5 \\
& $13: 35$ & $16: 50$ & $17: 35$ & 17.3 & 29.3 & 23.8 \\
\hline $3 / 16$ & $20: 00$ & $20: 45$ & $22: 30$ & 20.9 & 28 & 18.7 \\
\hline
\end{tabular}

\subsection{Development of Thermal Models}

The development of thermal models follows the typical machine learning pipeline, as shown in Figure 5. In this study, three different feature sets were used for developing the thermal models. The base feature set consists of indoor air temperature, indoor relative humidity and side face skin temperature. Since clothing plays an important role in thermal comfort, clothing surface temperature was selected as additional feature to construct feature set A. Moreover, since thermal comfort is also affected by temperature gradient, particularly in the transient conditions, side face skin temperature difference between two consecutive measurements was also selected as an additional feature to construct feature set $B$.

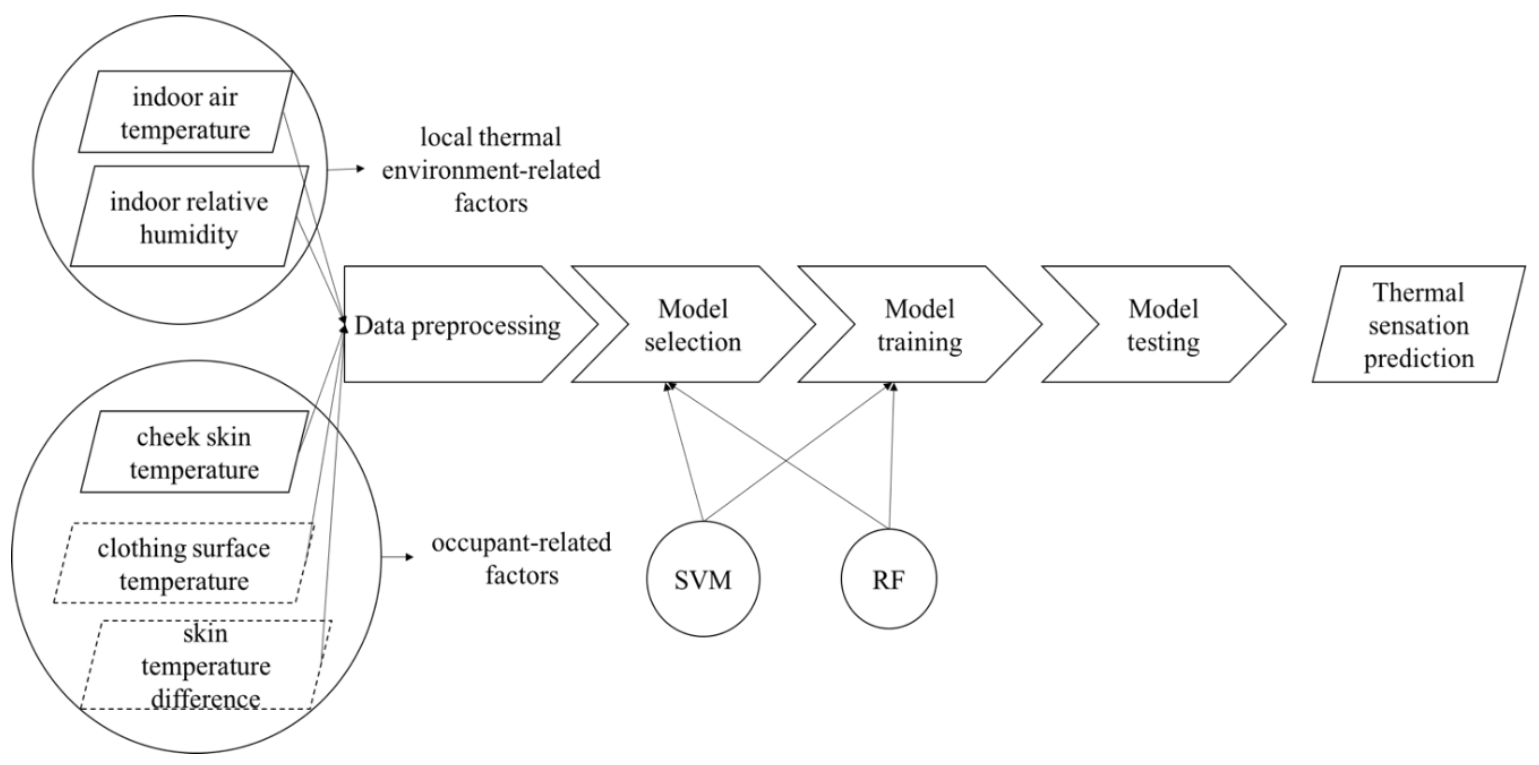

Figure 5. The development pipeline of thermal models.

As for the baseline, PMV was used to compare with the two classification models. However, several assumptions were set when calculating PMV. Firstly, since the mean radiant temperature (MRT) and indoor air velocity were not measured and the experimental environment avoided direct radiation, 
MRT was approximated with indoor air temperature when calculating PMV and the indoor air velocity was calculated as $0.1 \mathrm{~m} / \mathrm{s}$ since there was no draft risk. In addition, since the subjects were seated during the whole study, the metabolic rate was calculated as 1 met and there was no external work.

The whole dataset was split into two parts where $80 \%$ of data is the training set and $20 \%$ is the testing data. In addition, before training the best model, the hyper-parameters used in the best models were selected with 5-fold cross-validation, as shown in Table 4. Cross-validation is one of the common methods of model selection, which aims to find the model that gives the best prediction. The models fit on the training set is used to predict the test set. Usually, many such splits are used, and the results are averaged over splits [29]. In terms of classification algorithms, rather than neural network, SVM and RF were implemented for classification since they can perform well with a relatively small-scale dataset.

Table 4. The parameters of SVM and RF to be tuned with cross-validation.

\begin{tabular}{ccc}
\hline Algorithms & Parameters & Values \\
\hline SVM & Penalty parameter C & 1,10 \\
& Kernel type & Gaussian, linear \\
RF & Tree depth & 3,4 \\
& Number of trees & 50,100 \\
\hline
\end{tabular}

SVM can be used to develop supervised classification models with high-dimensional and non-linear data. Since the data is unlikely to be separated linearly, with SVM classification, soft margin usually performs better than hard margin [30]. Generally, penalty parameter of $C$ is used to control how soft of the margin can be selected with cross-validation. The Equations (1)-(3) show the optimization framework in SVM. Moreover, compared to quadratic programming to solve the optimization problem, kernelized SVM can be computed much more efficiently. The common kernel functions include linear kernel, polynomial kernel and Gaussian kernel.

$$
\text { Inputs : } S=\left\{\left(x_{1}, y_{1}\right),\left(x_{2}, y_{2}\right) \ldots,\left(x_{n}, y_{n}\right)\right\} \text {, where } x_{i} \in X_{\text {train }} \text { and } y_{i} \in Y_{\text {train }}
$$

Objective:

$$
\operatorname{argmin}_{w, \xi_{1}, \xi_{2} \ldots \xi_{n}}\|w\|^{2}+\sum_{i} \xi_{i}
$$

Subject to:

$$
\begin{gathered}
y_{i} w * x_{i} \geq 1-\xi_{i} \\
\xi_{i} \geq 0
\end{gathered}
$$

where $w$ is a weight vector, $\mathrm{C}$ is a penalty parameter controlling how much you want to avoid misclassifying each training example and $\xi_{i}$ is a slack variable indicating if the sample is misclassified. With larger $C$, the optimization will select smaller margin of the hyper plane while with smaller $C$, the optimization will select larger margin of the hyperplane.

Unlike SVM, RF is an ensemble learning method that averages the accuracy of a number of decision trees constructed with bagging algorithm at training time. However, random forest adds additional randomness to the model in that it looks for the most important feature among a random subset of features, a random subset of the whole dataset instead of all features, and the whole dataset while splitting the node. Compared to building deep decision trees to train high-dimensional data, owing to randomness, RF builds several smaller trees, thus suffering less from overfitting [31]. As a result, it generally can develop better model than decision trees.

\subsection{Assessment of Thermal Models}

The prediction results of SVM and RF were compared with PMV model and all the thermal models were evaluated based on recall, precision and F1 scores [32], which were calculated with the confusion 
matrix, as shown in Table 5. Recall score measures the ability of a model to find all correctly classified instances within a dataset, which is the ratio between true positives and all actual positives. Precision score describes how precise the model is out of the predicted results, which is the ratio between true positives and all predicted positives. Lastly, F1 score is used to seek a balance between recall and precision, which is a harmonic mean of recall and precision. Recall, precision and F1 scores can be computed with the following equations.

$$
\begin{aligned}
\text { Precision } & =\frac{\text { True positive }}{\text { True positive }+ \text { False positive }} \\
\text { Recall } & =\frac{\text { True positive }}{\text { True positive }+ \text { False negative }} \\
\text { F1 } & =2 * \frac{\text { Precision } * \text { Recall }}{\text { Precision }+ \text { Recall }}
\end{aligned}
$$

Table 5. Confusion matrix.

\begin{tabular}{ccc}
\hline & Predicted Negative & Predicted Positive \\
\hline Actual Negative & True negative & False positive \\
Actual Positive & False negative & True positive \\
\hline
\end{tabular}

\section{Result Analysis}

The raw data has a total of 775 instances, among which the male subject had 413 instances and the female subject had 362 instances. Moreover, Table 6 shows the number of instances collected from each thermal sensation. As shown in the table, the distribution is imbalanced where the neutral sensation is the majority vote.

Table 6. Data distribution over different sensations.

\begin{tabular}{cccccccc}
\hline & $\begin{array}{c}\text { Very Cold } \\
(\mathbf{- 3})\end{array}$ & $\begin{array}{c}\text { Cold } \\
\mathbf{( - 2 )}\end{array}$ & $\begin{array}{c}\text { Cool } \\
\mathbf{( - 1 )}\end{array}$ & $\begin{array}{c}\text { Neutral } \\
\mathbf{( 0 )}\end{array}$ & $\begin{array}{c}\text { Warm } \\
\mathbf{( 1 )}\end{array}$ & $\begin{array}{c}\text { Hot } \\
\mathbf{( 2 )}\end{array}$ & $\begin{array}{c}\text { Very Hot } \\
\mathbf{( 3 )}\end{array}$ \\
\hline Female & 3 & 16 & 29 & 168 & 73 & 58 & 15 \\
Male & 7 & 13 & 24 & 188 & 100 & 58 & 23 \\
\hline
\end{tabular}

\subsection{Relationships between Occupant-related Variables and Thermal Sensations}

In this study, two occupant-related variables were selected as features. One is side face skin temperature and the other is clothing surface temperature. Before developing the thermal models, the relationship between skin temperature and thermal sensation, as well as the relationship between clothing surface temperature and thermal sensation were investigated. Figure 6a-d show the relationships based on the data collected with the two subjects. As a result, for female subject, the correlation coefficient between skin temperature and thermal sensation is 0.747 while that between clothing surface temperature and thermal sensation is 0.768 . In addition, for male subject, the correlation coefficient between skin temperature and thermal sensation is 0.688 while that between clothing surface temperature and thermal sensation is 0.763 . Therefore, clothing surface temperature has a higher correlation with thermal sensation than skin temperature for both subjects. 


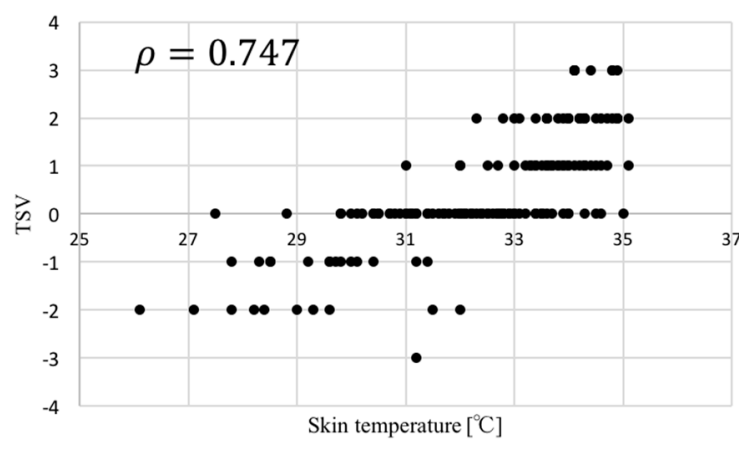

(a)

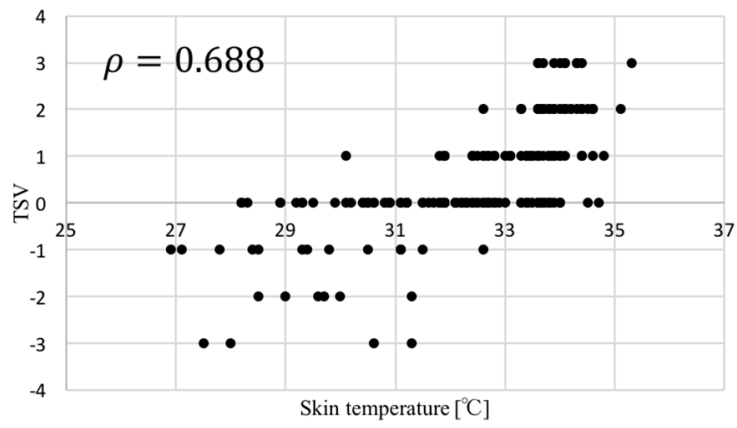

(c)

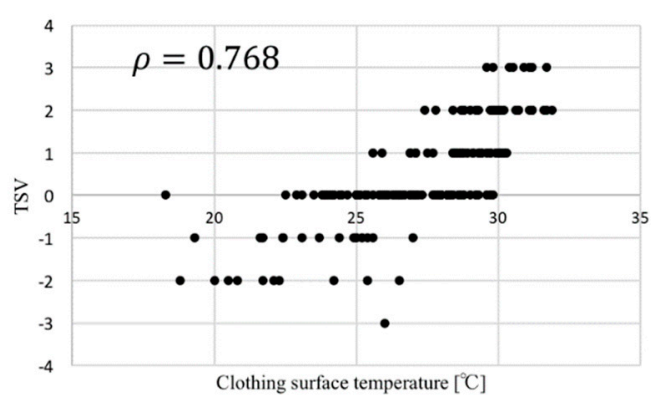

(b)

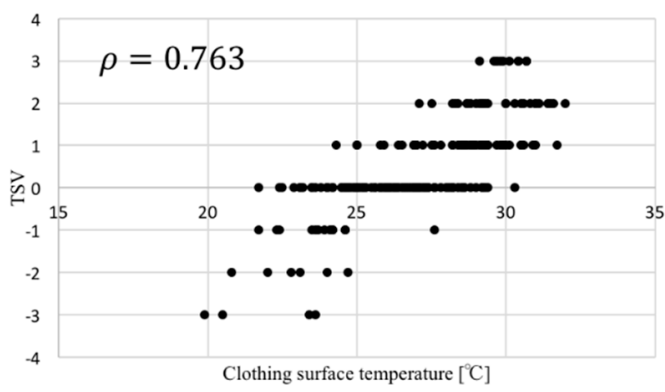

(d)

Figure 6. Relationships between thermal sensations and (a) skin temperature of female subject (b) clothing surface temperature of female subject (c) skin temperature of male subject (d) clothing surface temperature of male subject.

\subsection{Results of Thermal Models with Classification Algorithms}

Based on a 5-fold cross-validation with both female and male data, Table 7 shows the recall scores with cross-validation based on different parameter values of SVM and RF, respectively. As shown in the table, in terms of SVM model configuration, SVM with linear kernel has much better performance than Gaussian kernel with feature set A while there are few differences among all SVM configurations with the base feature set and feature set B for both subjects. Meanwhile, in terms of RF model configuration, there are few differences among all combinations of RF configurations and features sets. Since a larger penalty number will result in a slower SVM model and larger tree depth, and the number of trees will also result in a slower RF model, SVM with a linear kernel and penalty number of 1 were selected as the best model configuration SVM, and RF with maximum depth of 3 and 50 trees were selected as RF best model configuration.

Table 7. Best parameters selected with cross-validation.

\begin{tabular}{|c|c|c|c|c|c|c|}
\hline \multirow{3}{*}{ Model Configuration } & \multicolumn{6}{|c|}{ Average Recall Score } \\
\hline & \multicolumn{3}{|c|}{ Female } & \multicolumn{3}{|c|}{ Male } \\
\hline & Base & A & B & Base & A & B \\
\hline SVM, C = 1, kernel = linear & 0.65 & 0.98 & 0.65 & 0.62 & 0.96 & 0.57 \\
\hline SVM, C $=1$, kernel = Gaussian & 0.62 & 0.68 & 0.57 & 0.61 & 0.78 & 0.59 \\
\hline SVM, C $=10$, kernel $=$ linear & 0.62 & 0.98 & 0.6 & 0.6 & 0.96 & 0.6 \\
\hline SVM, C $=10$, kernel $=$ Gaussian & 0.62 & 0.82 & 0.52 & 0.6 & 0.88 & 0.58 \\
\hline RF, Max_depth $=3$, number of trees $=50$ & 0.6 & 0.96 & 0.6 & 0.68 & 0.95 & 0.59 \\
\hline RF, Max_depth $=3$, number of trees $=100$ & 0.6 & 0.96 & 0.59 & 0.58 & 0.96 & 0.61 \\
\hline RF, Max_depth $=4$, number of trees $=50$ & 0.63 & 0.97 & 0.59 & 0.57 & 0.99 & 0.62 \\
\hline RF, Max_depth $=4$, number of trees $=100$ & 0.62 & 0.99 & 0.59 & 0.6 & 0.98 & 0.6 \\
\hline
\end{tabular}


Tables 8 and 9 show the performance benchmark among the thermal models of the two subjects with the three feature sets trained by SVM, RF and PMV, respectively. As a result, for the female subject, precision, recall and F1 scores of PMV on test data are $48.6 \%, 48.6 \%$ and $48.3 \%$, respectively. Compared to the baseline of PMV, SVM and RF have better performances with all three different feature sets. Among the three feature sets, feature set A comprised of indoor air temperature, relative humidity, side face skin temperature and clothing surface temperature has $100 \%$ recall, precision and $\mathrm{F} 1$ score with SVM, which significantly outperforms the baseline. This may be because the effect of clothing insulation on thermal comfort has been taken into account since the dynamic clothing insulation can be estimated with clothing surface temperature and skin temperature [30], and the causal effect analysis in Section 3.1 also illustrates the clothing surface temperature has a higher correlation with thermal sensations than the skin temperature. In addition, SVM performs better than RF with all three feature sets.

Table 8. Performance benchmark among the thermal models of the female subject.

\begin{tabular}{cccccccccc}
\hline \multirow{2}{*}{ Algorithm } & \multicolumn{3}{c}{ Base Feature Set } & \multicolumn{3}{c}{ Feature Set A } & \multicolumn{3}{c}{ Feature Set B } \\
\cline { 2 - 9 } & P (\%) & R (\%) & F1 (\%) & P (\%) & R (\%) & F1 (\%) & P (\%) & R (\%) & F1 (\%) \\
\hline SVM & 62.3 & 62.9 & 62 & 100 & 100 & 100 & 69.2 & 64.7 & 65.1 \\
RF & 57.7 & 48.6 & 48.6 & 91.6 & 91.4 & 91 & 49 & 47 & 46.3 \\
PMV & 48.6 & 48.6 & 48.3 & 48.6 & 48.6 & 48.3 & 48.6 & 48.6 & 48.3 \\
\hline
\end{tabular}

SVM: support vector machine; RF: random forest; PMV: predicted mean vote.

Table 9. Performance benchmark among the thermal models of the male subject

\begin{tabular}{cccccccccc}
\hline \multirow{2}{*}{ Algorithm } & \multicolumn{3}{c}{ Base Feature Set } & \multicolumn{3}{c}{ Feature Set A } & \multicolumn{3}{c}{ Feature Set B } \\
\cline { 2 - 9 } & P (\%) & R (\%) & F1 (\%) & P (\%) & R (\%) & F1 (\%) & P (\%) & R (\%) & F1 (\%) \\
\hline SVM & 33.5 & 42.5 & 35.8 & $\mathbf{9 7 . 5}$ & $\mathbf{9 5}$ & $\mathbf{9 6 . 1}$ & 32.1 & 43.6 & 34.5 \\
RF & 28.8 & 40 & 33.1 & 90.4 & 92.5 & 91.4 & 26.8 & 38.5 & 30.7 \\
PMV & 35 & 33 & 31.8 & 35 & 33 & 31.8 & 35 & 33 & 31.8 \\
\hline
\end{tabular}

SVM: support vector machine; RF: random forest; PMV: predicted mean vote.

For the male subject, precision, recall and F1 score of PMV on test data is only $35 \%, 33 \%$ and $31.8 \%$, respectively. Moreover, even if outperforming the baseline with the recall score, both SVM and RF have much worse performances in predicting thermal sensation of the male subject with the base feature set and the feature set $B$ than those of female subject. This indicates individual differences with respect to thermal comfort such as gender, thus the personalized heating and cooling system being important to develop. However, for the feature set A, SVM still achieves high precision, recall and f1 scores which indicates that SVM can be the best classifier with the feature set consisting of indoor air temperature, relative humidity, skin temperature and clothing surface temperature for both the female and male subject. Therefore, such a combination is recommended to be the thermal model for real-time prediction for automatic personalized heating and cooling system in open-plan offices.

Moreover, Figures 7 and 8 show the normalized confusion matrices on test data with the three feature sets for the two subjects by using SVM and RF, respectively. The normalization equation is shown below:

$$
x_{n o r m \_i j}=\frac{x_{i j}}{\sum_{k=1}^{k=m} x_{i k}}
$$

where $x_{n o r m} \_i j$ is the normalized value of cell $x_{i j}$, which is the element in the $i$ th row and $j$ th column. In addition, $m$ is the number of the column of the confusion matrix. 


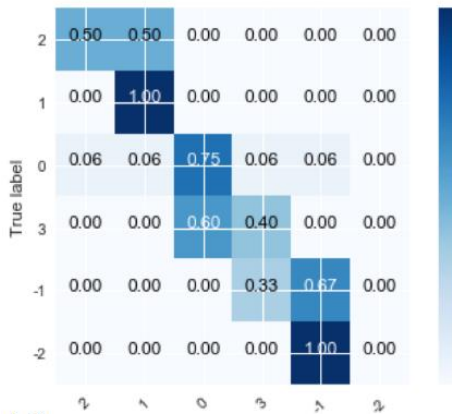

(a)

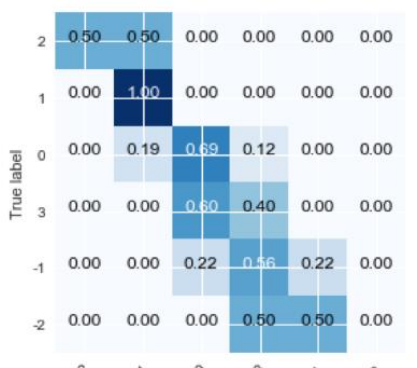

(d)
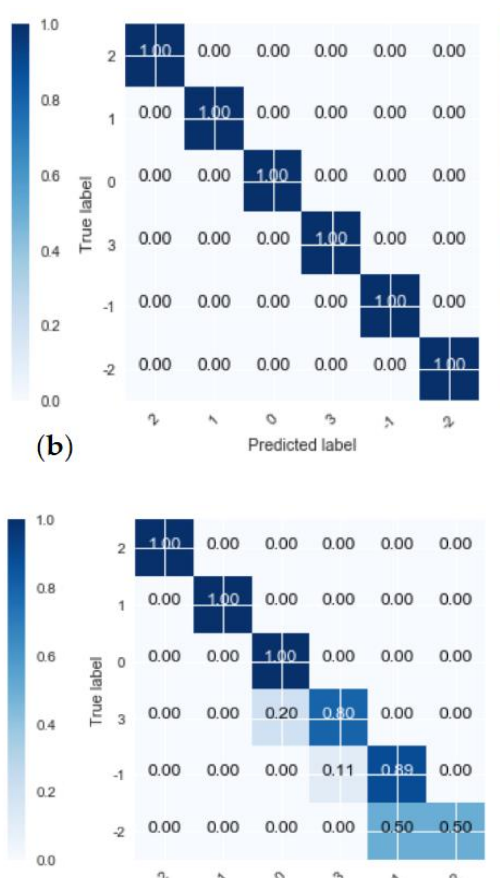

(e)

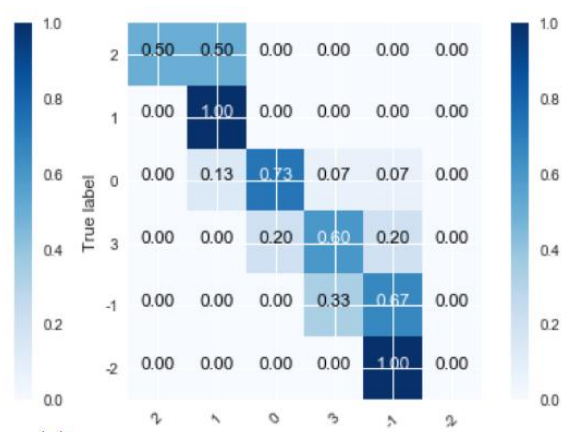

(c)

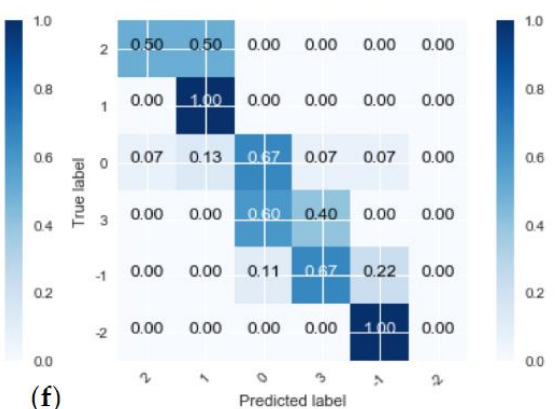

(f)

Figure 7. Normalized confusion matrix of the female subject. (a) Base Feature set with SVM, (b) Feature set A with SVM, (c) Feature set B with SVM, (d) Base Feature set with RF, (e) Feature set A with RF, (f) Feature set B with RF.

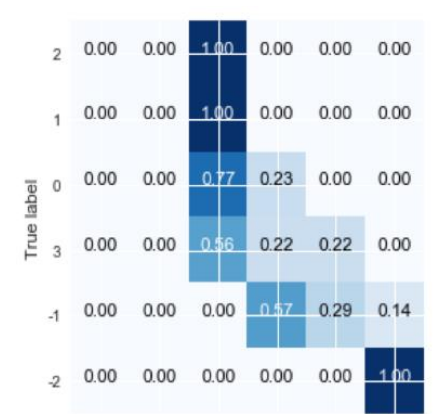

(a)

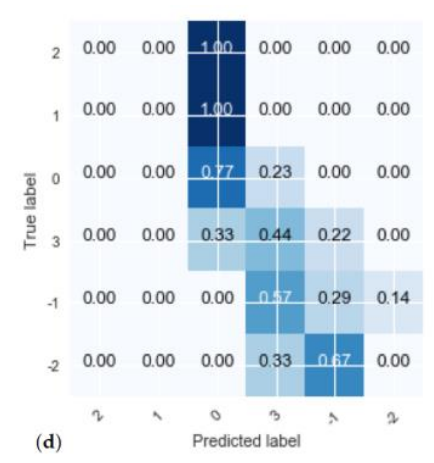

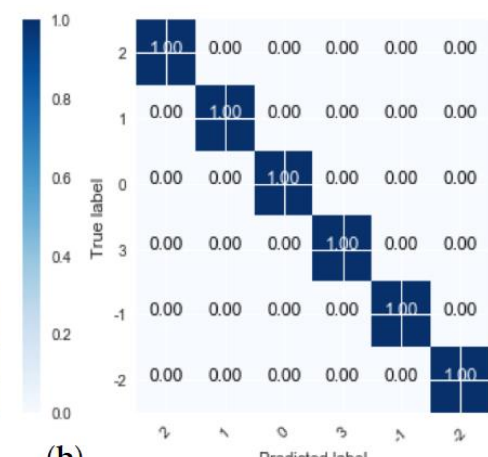

(b)

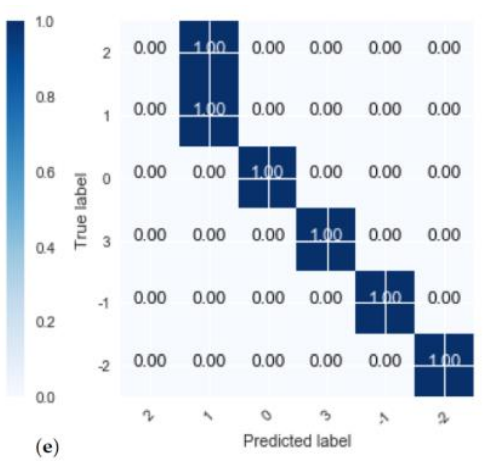

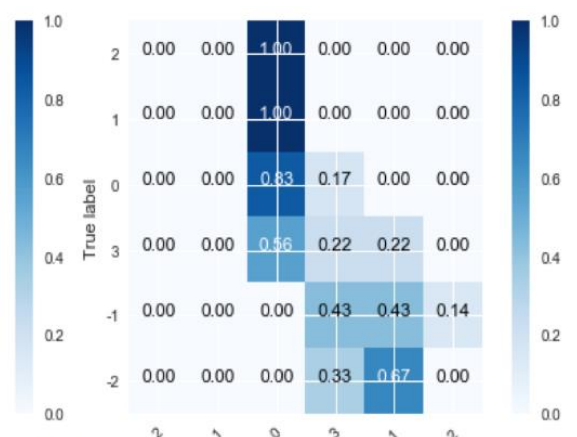

(c)

Figure 8. Normalized confusion matrix of the male subject. (a) Base Feature set with SVM, (b) Feature set A with SVM, (c) Feature set B with SVM, (d) Base Feature set with RF, (e) Feature set A with RF, (f) Feature set B with RF.

As to the female subject, the test data with neutral label has the highest accuracy for each feature set. With both SVM and RF, for base feature set, the test data with cold $(-2)$ label has the lowest accuracy 
while for feature set B, the test data with cool (-1) label has the lowest accuracy. As to male subject, same as female subject, the test data with neutral label has the highest accuracy for each feature set. Moreover, as shown in Table 5, the number of uncomfortable sensations is much smaller than that of neutral sensation. Therefore, it is more difficult to predict uncomfortable thermal sensations, including cold/hot (-2/2) and very cold/very hot (-3/3) than neutral sensation. However, as shown in the figures, SVM performs better in predicting uncomfortable thermal sensations than RF for both subjects.

\section{Discussions}

With profound studies on the relationship between building energy performance and human dimensions, i.e., that occupant behaviors actually play a significant role in energy consumption in the buildings [33], the occupant-responsive HVAC system has been seen as future trend for developing intelligent building systems due to the outstanding performance of energy savings and occupant comfort improvements. Moreover, compared to theoretical physical models, the data-driven approaches to studying building system and thermal comfort have emerged to mitigate the difficulty in developing complex models and still maintain high accuracy [8,11-20,23-26,34]. Moreover, compared to wearable sensing techniques, a non-intrusive sensing technique has drawn more attention for occupant-related factors related to thermal comfort. Therefore, this paper proposes to use the non-intrusive infrared camera in combination with temperature and humidity sensors to infer individual thermal comfort in real-time with two classification algorithms of SVM and RF.

Compared to existing methods, besides skin temperature, the proposed method also takes clothing surface temperature and skin temperature difference into consideration. Moreover, unlike ASHRAE RP884 where only a few instances were collected for each individual, the proposed experimental study collected over 300 instances for each individual so that the amount of data is enough for those classification algorithms. Additionally, besides using machine learning algorithms for thermal comfort inference like existing literature, this study also conducted a causal analysis with correlation coefficient to interpret the machine learning classification results. The results have shown the model with the feature set consisting of indoor air temperature, indoor relative humidity, side face skin temperature and clothing surface temperature perform best for both subjects. This can be explained by the fact that the correlation coefficient between clothing surface temperature and thermal sensation is higher than that between skin temperature and thermal sensation. Additionally, the model performance with respect to the female subject is much better than that of the male subject with the base feature set and the feature set $B$, which reveals individual differences and indicates it is necessary to update the individual thermal models with more data in real-time when adapting new users.

Moreover, besides office buildings, the proposed non-intrusive sensing system could also be applied to the air-conditioning system control during the night. During the period when occupants are asleep, whether the temperature set-point of the air-conditioning system is comfortable or not will affect sleep quality $[35,36]$. However, since it is unlikely for occupants to manually adjust set-points of the system during sleep, the non-intrusive sensing system could measure thermal environment and skin temperature without interruptions. In addition, the sensing system could also be applied to vehicle cabins [37]. Due to the much smaller size of the inner space, the non-intrusive sensing system could be embedded into the HVAC system where it does not affect the driver's views. However, since the current infrared camera is expensive to use, it may not be ideal for the real application in terms of thermal comfort. Therefore, more cost-effective sensing technique shall be investigated. AMG8833 is an $8 \times 8$ infrared thermal array used for occupancy detection where each pixel measures a surface temperature. Under normal operating temperature and the measured surface temperature, a calibrated AMG8833 has the resolution of $0.1^{\circ} \mathrm{C}$ and the accuracy of $+/-0.5^{\circ} \mathrm{C}$ [38]. Since AMG8833 is not only much more cost-effective than the infrared camera but also more easily integrated with temperature and humidity sensor such as DHT22, it has great potential to be used as a non-intrusive sensing system for the personalized HVAC system in different scenarios such as open-plan office buildings, residential buildings and vehicles. 


\section{Conclusions}

This paper proposes a non-intrusive sensing technique, which consists of temperature, humidity sensor called DHT22, and an infrared camera named FLIR B8400. In order to improve prediction performance of thermal comfort, individual thermal models for female subject and male subject were developed with SVM and RF based on a six day experimental study conducted in an open-plan office in Shanghai.

A total of three different feature sets were selected to develop individual thermal models with the labels based on 7-point thermal sensation scale. As a result, all proposed feature sets have achieved much better performances than the baseline of the PMV model. In addition, the model trained with the feature set consisting of indoor air temperature, indoor relative humidity, side face skin temperature and clothing surface temperature, and with linear kernel SVM has achieved the best performance. The precision, recall and f1 scores of the best model was $100 \%$ on test data of female subjects and $97.5 \%$, $96.1 \%$ and $95 \%$ on that of male subjects, respectively. Besides classification, the causal analysis has also shown that clothing surface temperature has a higher correlation coefficient with thermal sensations than side face skin temperature for both subjects, which explains why the individual thermal models with feature sets containing clothing surface temperature have the best performance. Last but not least, the individual difference in thermal comfort indicates the importance of personalized heating and cooling systems.

Moreover, the proposed sensing system could be improved further by using smaller-sized infrared thermal array such as AMG8833 to realize large-scale real deployment in open-plan office buildings. In future, the personalized cooling system could also be applied to various scenarios besides open-plan offices, such as sleep mode and driving mode to improve thermally comfortable indoor environments and energy performance.

Author Contributions: The following statements should be used conceptualization, S.L.; methodology, S.L. and S.W.; software, S.L. and S.W.; validation, S.L. and W.W.; formal analysis, S.L., S.W. and W.W.; investigation, S.L., S.W. and W.W.; resources, S.L.; data curation, S.L.; writing-original draft preparation, S.L.; writing-review and editing, W.W. and E.C.H.; visualization, S.L. and W.W.; supervision, E.C.H.; project administration, S.L. and E.C.H.; funding acquisition, S.L. and E.C.H.

Funding: This research received no external funding.

Acknowledgments: Siliang Lu is sponsored by Chinese Scholarship Council and this work is partially supported by CMU Graduate Small Project Help (GuSH) Research Grants. Moreover, many thanks to Jie Shi for providing the test rooms.

Conflicts of Interest: The authors declare no conflict of interest.

\section{References}

1. Bratman, G.N.; Hamilton, J.P.; Daily, G.C. The impacts of nature experience on human cognitive function and mental health. Ann. N. Y. Acad. Sci. 2012, 1249, 118-136. [CrossRef]

2. ASHRAE. Standard 55-2013. Thermal Environmental Conditions for Human Occupancy, 12; ASHRAE: Atlanta, GA, USA, 2013.

3. Roelofsen, P. Evaluation of draught in surgical operating theatres: Proposed revision to (NEN)-EN-ISO-7730. J. Facil. Manag. 2011, 9, 64-70. [CrossRef]

4. Fanger, P.O. Thermal comfort. Analysis and applications in environmental engineering. Thermal comfort. Analysis and applications in environmental engineering. SAJE J. 1970. [CrossRef]

5. De Dear, R.; Brager, G.S. Developing an Adaptive Model of Thermal Comfort and Preference; UC Berkeley: Berkeley, CA, USA, 1998.

6. Lee, S.; Bilionis, I.; Karava, P.; Tzempelikos, A. A Bayesian approach for probabilistic classification and inference of occupant thermal preferences in office buildings. Build. Environ. 2017, 118, 323-343. [CrossRef]

7. Auffenberg, F.; Stein, S.; Rogers, A. A personalized thermal comfort model using a Bayesian network. In Proceedings of the 24th International Conference on Artificial Intelligence, Buenos Aires, Argentina, 25-31 July 2015. 
8. Kim, J.; Zhou, Y.; Schiavon, S.; Raftery, P.; Brager, G. Personal comfort models: Predicting individuals' thermal preference using occupant heating and cooling behavior and machine learning. Build. Environ. 2018, 129, 96-106. [CrossRef]

9. Parsons, K. Human Thermal Environments: The Effects of Hot, Moderate, and Cold Environments on Human Health, Comfort, and Performance; CRC Press: Boca Raton, FL, USA, 2014.

10. Shapiro, Y.; Epstein, Y. Environmental physiology and indoor climate-Thermoregulation and thermal comfort. Energy Build. 1984, 7, 29-34. [CrossRef]

11. Liu, W.; Lian, Z.; Deng, Q. Use of mean skin temperature in evaluation of individual thermal comfort for a person in a sleeping posture under steady thermal environment. Indoor Built Environ. 2015, 24, 489-499. [CrossRef]

12. Choi, J.H. CoBi: Bio-Sensing Building Mechanical System Controls for Sustainably Enhancing Individual Thermal Comfort. Ph.D. Thesis, Carnegie Mellon University, Pittsburgh, PA, USA, 2010.

13. Sim, S.Y.; Koh, M.J.; Joo, K.M.; Noh, S.; Park, S.; Kim, Y.H.; Park, K.S.; Sabatini, A.M. Estimation of Thermal Sensation Based on Wrist Skin Temperatures. Sensors 2016, 16, 420. [CrossRef]

14. Li, W.; Zhang, J.; Zhao, T.; Liang, R. Experimental research of online monitoring and evaluation method of human thermal sensation in different active states based on wristband device. Energy Build. 2018, 173, 613-622. [CrossRef]

15. Ghahramani, A.; Castro, G.; Karvigh, S.A.; Becerik-Gerber, B. Towards unsupervised learning of thermal comfort using infrared thermography. Appl. Energy 2018, 211, 41-49. [CrossRef]

16. Huang, C.-C.J.; Yang, R.; Newman, M.W. The potential and challenges of inferring thermal comfort at home using commodity sensors. In Proceedings of the 2015 ACM International Joint Conference on Pervasive and Ubiquitous Computing, Osaka, Japan, 7-11 September 2015; pp. 1089-1100.

17. Dai, C.; Zhang, H.; Arens, E.; Lian, Z. Machine learning approaches to predict thermal demands using skin temperatures: Steady-state conditions. Build. Environ. 2017, 114, 1-10. [CrossRef]

18. Ghahramani, A.; Castro, G.; Becerik-Gerber, B.; Yu, X. Infrared thermography of human face for monitoring thermoregulation performance and estimating personal thermal comfort. Build. Environ. 2016, 109, 1-11. [CrossRef]

19. Hasan, M.H.; Alsaleem, F.; Rafaie, M. Sensitivity study for the PMV thermal comfort model and the use of wearable devices biometric data for metabolic rate estimation. Build. Environ. 2016, 110, 173-183. [CrossRef]

20. Abdallah, M.; Clevenger, C.; Vu, T.; Nguyen, A. Sensing Occupant Comfort Using Wearable Technologies. In Proceedings of the Construction Research Congress, San Juan, Puerto Rico, 31 May-2 June 2016; pp. 940-950.

21. Osornio-Rios, R.A.; Antonino-Daviu, J.A.; Romero-Troncoso, R.D.J.; Osornio-Rios, R.A.A. Recent Industrial Applications of Infrared Thermography: A Review. IEEE Trans. Ind. Inform. 2019, 15, 615-625. [CrossRef]

22. Nardi, I.; Lucchi, E.; De Rubeis, T.; Ambrosini, D. Quantification of heat energy losses through the building envelope: A state-of-the-art analysis with critical and comprehensive review on infrared thermography. Build. Environ. 2018, 146, 190-205. [CrossRef]

23. Tanda, G. Skin temperature measurements by infrared thermography during running exercise. Exp. Therm. Fluid Sci. 2016, 71, 103-113. [CrossRef]

24. Ranjan, J.; Scott, J. ThermalSense: Determining dynamic thermal comfort preferences using thermographic imaging. In Proceedings of the 2016 ACM International Joint Conference on Pervasive and Ubiquitous Computing, Heidelberg, Germany, 12-16 September 2016; pp. 1212-1222.

25. Han, D.; Li, R.; Wang, F.; Sun, Z.; Moon, S.; Gong, Z.; Yu, W.; Zhang, Y. Study on Indoor Thermal Environment Control Based on Thermal Sensation Prediction. Procedia Eng. 2017, 205, 3072-3079. [CrossRef]

26. Li, D.; Menassa, C.C.; Kamat, V.R. Non-intrusive interpretation of human thermal comfort through analysis of facial infrared thermography. Energy Build. 2018, 176, 246-261. [CrossRef]

27. Pedregosa, F.; Varoquaux, G.; Gramfort, A.; Michel, V.; Thirion, B.; Grisel, O.; Blondel, M.; Prettenhofer, P.; Weiss, R.; Dubourg, V.; et al. Scikit-learn: Machine Learning in Python. J. Mach. Learn. Res. 2011, 12, 2825-2830.

28. Auliciems, A.; Szokolay, S.V. Thermal Comfort. PLEA. 1997. Available online: http://www.labeee.ufsc.br/ sites/default/files/disciplinas/Szokolay\%20e\%20Auliciems,\%201997.pdf (accessed on 20 April 2019).

29. Kohavi, R. A study of cross-validation and bootstrap for accuracy estimation and model selection. IJCAI 1995, 14, 1137-1145.

30. Smola, A.J.; Schölkopf, B. A tutorial on support vector regression. Stat. Comput. 2004, 14, 199-222. [CrossRef] 
31. Liaw, A.; Wiener, M. Classification and regression by random Forest. $R$ News 2002, 2, 18-22.

32. Goutte, C.; Gaussier, E. A probabilistic interpretation of precision, recall and F-score, with implication for evaluation. In European Conference on Information Retrieval; Springer: Berlin/Heidelberg, Germany, 2005; pp. 345-359.

33. D'Oca, S.; Hong, T.; Langevin, J. The human dimensions of energy use in buildings: A review. Renew. Sustain. Energy Rev. 2018, 81, 731-742. [CrossRef]

34. Smarra, F.; Jain, A.; De Rubeis, T.; Ambrosini, D.; D’Innocenzo, A.; Mangharam, R. Data-driven model predictive control using random forests for building energy optimization and climate control. Appl. Energy 2018, 226, 1252-1272. [CrossRef]

35. Lan, L.; Zhai, Z. (John); Lian, Z. A two-part model for evaluation of thermal neutrality for sleeping people. Build. Environ. 2018, 132, 319-326. [CrossRef]

36. Lan, L.; Tsuzuki, K.; Liu, Y.; Lian, Z. Thermal environment and sleep quality: A review. Energy Build. 2017, 149, 101-113. [CrossRef]

37. Lee, J.-H.; Kim, Y.-K.; Kim, K.-S.; Kim, S.; Lamberti, F. Estimating Clothing Thermal Insulation Using an Infrared Camera. Sensors 2016, 16, 341. [CrossRef] [PubMed]

38. Abbas, M. Frequently Asked Questions: Infrared Grideye Sensor; Panasonic Inc., 2015. Available online: https://cdn.sparkfun.com/assets/2/0/3/f/b/faqs_grideye_v1.0.pdf (accessed on 20 April 2019).

(C) 2019 by the authors. Licensee MDPI, Basel, Switzerland. This article is an open access article distributed under the terms and conditions of the Creative Commons Attribution (CC BY) license (http://creativecommons.org/licenses/by/4.0/). 